\title{
ADAM33 protein expression and the mechanics of airway smooth muscle cells are highly correlated in ovalbumin-sensitized rats
}

\author{
FENG LIN $^{1}$, AIJING SONG ${ }^{2}$, JIAMIN WU ${ }^{1}$, XUEMEI JIANG ${ }^{1}$, JIAOYUE LONG ${ }^{1}$, \\ JUN CHEN $^{1}$, YIYUAN DUAN ${ }^{1}$, YANLING SHI $^{1}$ and LINHONG DENG ${ }^{1,3}$ \\ ${ }^{1}$ Key Laboratory of Biorheological Science and Technology, Ministry of Education, Bioengineering College, \\ Chongqing University, Chongqing 400044; ${ }^{2}$ Guangdong Provincial Institute of Sports Science, \\ Guangzhou, Guangdong 510663, P.R. China
}

Received February 14, 2013; Accepted July 15, 2013

DOI: $10.3892 / \mathrm{mmr} .2013 .1621$

\begin{abstract}
A disintegrin and metalloproteinase 33 (ADAM33) has been identified as an asthma susceptibility gene; however, the role of ADAM33 in the pathogenesis and progression of asthma remains to be elucidated. As ADAM33 is predominantly expressed in airway smooth muscle cells (ASMCs), it is feasible to investigate whether ADAM33 protein expression is correlated with ASMC mechanics that are ultimately responsible for airway hyperresponsiveness in asthma. To determine this, Sprague Dawley rats were sensitized with ovalbumin (OVA) for up to 12 weeks to simulate asthma symptoms. Subsequently, ASMCs were isolated from the rats and cultured in vitro. The protein expression of ADAM33 and cytoskeletal proteins (including F-actin and vinculin), cell stiffness and contractility, as well as traction force were measured. The results demonstrated that compared with the non-sensitized rats, the protein expression of ADAM33 in ASMCs from the OVA-sensitized rats increased in a time-dependent manner, reaching a maximum level at 4 weeks of sensitization and gradually subsiding as OVA sensitization continued $(\mathrm{P}<0.001)$. The cell stiffness, traction force and expression of vinculin and F-actin changed similarly, resulting in a positive correlation with ADAM33 protein expression (Pearson's correlation coefficient, 0.864, 0.716, 0.774 and 0.662, respectively; $\mathrm{P}=0.1-0.3$ ). The in vivo results of OVA-induced ADAM33 protein expression and its association with the mechanics of ASMCs
\end{abstract}

Correspondence to: Professor Linhong Deng, Key Laboratory of Biorheological Science and Technology, Ministry of Education, and Bioengineering College, Chongqing University, 174 Shapingzhengjie Street, Chongqing 400044, P.R. China

E-mail: denglh@cqu.edu.cn

Present address: ${ }^{3}$ Institute of Biomedical Engineering and Health Sciences, Changzhou University, 1 Gehulu Road, Changzhou, Jiangsu 213164, P.R. China

Key words: ADAM33, airway smooth muscle cell, cell stiffness, traction force, asthma suggested that ADAM33 is a mediator of ASMC dysfunction in asthma, and may provide a rationale for the therapeutic targeting of ADAM33 in the treatment of asthma.

\section{Introduction}

Asthma is a common chronic airway disease that is characterized by airway inflammation, airway tissue remodeling and airway hyperresponsiveness (AHR) $(1,2)$. Although the symptoms of asthma are largely controllable, the disease is often not completely curable at present. This is generally attributed to the absence of a complete understanding of the mechanism(s) of asthma pathogenesis. Previously, complex interactions between multiple genetic and environmental factors contributing to the pathogenesis of asthma have been demonstrated (3). Thus, a full investigation of the genes associated with asthma is required to ascertain their roles in mediating airway pathologies.

In the present study, one particular gene of interest, a disintegrin and metalloproteinase 33 (ADAM33), was observed for its correlation with the mechanics of airway smooth muscle cells (ASMCs) in ovalbumin (OVA)-sensitized rat models of asthma. In human studies, ADAM33 has been identified as an asthma susceptibility gene $(1,4-8)$. Subsequently, the correlations between the ADAM33 gene and airway inflammation and airway tissue remodeling have been demonstrated $(9,10)$. However, the correlation between the ADAM33 protein and AHR that is equally, if not more important than airway inflammation and tissue remodeling in asthma, remains to be studied. AHR is determined by the mechanics of ASMCs and the ADAM33 protein is predominantly expressed in ASMCs $(6,10,11)$. Thus, it is feasible that in asthma, the protein level of ADAM33 is altered, which may correlate with the mechanics of ASMCs and contribute to the induction of asthma development.

To test this hypothesis, Sprague Dawley (SD) rats were sensitized with OVA for up to 12 weeks to model chronic asthma. The protein expression of ADAM33 was then measured, along with the stiffness and contractility, traction force generation and cytoskeletal structure of the ASMCs obtained from the animal models at different time points of sensitization. The results demonstrated that the protein expres- 
sion of ADAM33 in the ASMCs of the sensitized rats increased compared with that of the controls. However, the increase peaked at 4 weeks of sensitization, and gradually declined as sensitization continued. Notably, the majority of mechanical properties appeared to change similarly throughout the sensitization period, resulting in a positive correlation between the protein expression of ADAM33 and the stiffness, traction force generation, and expression of viculin and F-actin. This implied that ADAM33 protein expression was correlated with the mechanics of ASMCs, and that the two may be concurrently involved in the pathogenesis and progression of asthma.

\section{Materials and methods}

Animal models and ASMC culture. Male SD rats (purchased from Chonqing Medical School, Chonqing, China) were sensitized by OVA to simulate asthma symptoms, following a standard protocol as described previously $(12,13)$. The study was approved by the Animal Investigational Committee of Chongqing Medical School, Chongqing, China. In brief, the rats were injected with $1 \% \mathrm{OVA}$ and $10 \% \mathrm{Al}(\mathrm{OH})_{3}$ in $\mathrm{NaCl}$ solution on days 1 and 7. From day 14, the rats were sensitized by OVA three times per week for 4,6 or 12 weeks, to mimic chronic asthma at different stages (data not shown). Rats recieving the same treatment schedule, but with saline instead of OVA, were used as controls. At given time points of sensitization (at 4,6 and 12 weeks), primary ASMCs were isolated from the SD rats and cultured in vitro according to the method described previously (14). In this study, all the cells used were between passages 2 to 5 .

Immunoblotting for ADAM33 protein. Protein expression of ADAM33 was assessed by western blot analysis as described previously $(1,9)$. Briefly, when the ASMCs grew to $90 \%$ confluence, they were lysed with $1 \mathrm{X}$ loading buffer (Beyotime Biotech, Jiangsu, China) to extract the total proteins. Subsequently, the proteins from each group, together with $\beta$-tubulin as the reference marker, were loaded in an equal volume of solution onto $10 \%$ sodium dodecyl sulfate-polyacrylamide gel electrophoresis (SDS-PAGE) gels. Western blot analysis was performed using a specific primary antibody for ADAM33 protein [AV49937; Sigma-Aldrich, St. Louis, MO, USA; diluted to $1: 200$ in $1 \%$ bovine serum albumin (BSA)] and $\beta$-tubulin (Cell Signaling Technology, Inc., Beverly, MA, USA) overnight at $4^{\circ} \mathrm{C}$. Subsequently, detection was performed using the corresponding horseradish peroxidase-conjugated secondary antibodies (Wuhan Boster, Biological Technology, Ltd., Wuhan, China). The protein expression of ADAM33 and $\beta$-tubulin was detected using an enhanced chemiluminescence kit (Beyotime Biotech, Jiangsu, China), and the ratio of the protein expression of ADAM33 to that of $\beta$-tubulin was used for quantitative assessment.

Assessment of ASMC stiffness and contractility. To assess ASMC mechanical properties, the cell stiffness and contractility were measured by optical magnetic twisting cytometry (OMTC), as previously described $(13,15)$. Briefly, ASMCs were seeded into 96-well cell culture dishes (Costar, Corning, Inc., NY, USA) coated with collagen (type I, Sigma-Aldrich) at a density of 20,000 cells/well and cultured for $\geq 12 \mathrm{~h}$ in serum-free medium. Following this, ferrimagnetic beads (diameter, $4.5 \mu \mathrm{m}$, provided by Dr. J. J. Fredberg of Harvard School of Public Health, Boston, MA, USA) pre-coated with synthetic Arginine-Glycine-Aspartic acid (RGD; $50 \mu \mathrm{g}$ peptide/mg beads; Integra Life Sciences, Plainsboro Township, NJ, USA) were added and the cells were incubated for $20 \mathrm{~min}$ to allow specific binding to the integrin receptors on the cell surface (approximately one or two beads per cell). The beads were then magnetized horizontally and twisted in an oscillatory magnetic field at a frequency of $0.1-100 \mathrm{~Hz}$. The cell stiffness $\left(G^{\prime}\right)$ was measured as the ratio between the applied magnetic torque and bead displacement $(\mathrm{Pa} / \mathrm{nm})$.

The change in cell stiffness in response to stimulation by contractile agonist, such as $\mathrm{KCl}$, was observed in the ASMCs. ASMC stiffness was measured at a constant oscillation frequency $(0.3 \mathrm{~Hz})$ for up to $60 \mathrm{sec}$. Following this, the contractile agonist, $\mathrm{KCl}(80 \mathrm{mM}$; iso-osmotic in this case), was immediately added to the cells. In response to $\mathrm{KCl}$, the cells contracted, and thus, the cell stiffness increased in $\sim 1 \mathrm{~min}$. The ratio between the cell stiffness following the addition of $\mathrm{KCl}$ and prior to the addition of $\mathrm{KCl}$ was defined as the contractility of the ASMCs (16).

Assessment of traction force generation by ASMCs. Traction force generated by ASMCs was measured using an elastic polyacrylamide gel substrate embedded with $0.2-\mu \mathrm{m}$ diameter fluorescence beads (F8811; Invitrogen Life Technologies, Carlsbad, CA, USA) as described previously $(17,18)$. Briefly, the polyacrylamide gel was prepared by adjusting the ratio of $40 \%$ acrylamide and $2 \%$ bis-acrylamide to produce a gel with a Young's modulus of $4 \mathrm{kPa}$. The prepared gel was placed in a cell culture dish with a glass bottom (to enable microscopy) and allowed to polymerize, forming an elastic substrate with a diameter of $\sim 18 \mathrm{~mm}$ and a thickness of $0.1 \mathrm{~mm}$. Following gel polymerization, the substrate was activated by sulfo-SANPAH (Pierce Biotechnology, Inc., Rockford, IL, USA) and coated with $0.2 \mathrm{mg} / \mathrm{ml}$ collagen solution (type I) overnight at $4^{\circ} \mathrm{C}$. The following day, the substrate was hydrated with $2 \mathrm{ml}$ Dulbecco's modified Eagle's medium/F-12 and incubated at $37^{\circ} \mathrm{C}$ and $5 \%$ $\mathrm{CO}_{2}$ for $\geq 24 \mathrm{~h}$ prior to use.

Cells were seeded onto the polyacrylamide gel substrate at a density of $\sim 2000-5000$ cells/well, incubated for $12 \mathrm{~h}$ to allow the cells to attach to the substrate and cultured for $\geq 12 \mathrm{~h}$ in serum-free medium. Single ASMCs were imaged with phase contrast by an inverted optical microscope (Leica DMI6000B; Leica Microsystems CMS GmbH, Wetzlar, Germany) and then detached from the substrate by trypsinization. Fluorescence imaging of the fluorescent beads embedded in the gel substrate was conducted with the inverted optical microscope prior to and following cell detachment. The traction force generated by the cells was subsequently computed by the displacement field of the elastic substrate as measured by the positions of the embedded fluorescent beads prior to and following cell detachment (18).

Assessment of the ASMC cytoskeletal structure. To assess the cytoskeletal structure, ASMCs were labeled with fluorescent probes for vinculin and F-actin, and analyzed by confocal microscopy. Vinculin and F-actin are essential components of the focal adhesions and the microfilament cytoskeleton, 
respectively, and are therefore commonly used to visualize and evaluate the structure of the cytoskeleton $(17,19)$. The ASMCs were fixed with $4 \%$ formaldehyde for $\sim 20 \mathrm{~min}$, washed with phosphate-buffered saline (PBS) and permeabilized with $0.2 \%$ Triton X-100 in PBS for 5 min at room temperature. Following washing with PBS, cells were treated with a blocking solution (1\% BSA) for $30 \mathrm{~min}$ at room temperature.

To label vinculin with a fluorescent probe, the cells were incubated with monoclonal anti-vinculin antibody (Abcam, Cambridge, UK; 1:200 dilution in 1\% BSA) overnight at $4^{\circ} \mathrm{C}$. The cells were washed with PBS, and further incubated with the secondary antibody, rhodamine labeled goat anti-mouse IgG (ProteinTech, Chicago, IL, USA; 1:100 dilution in 1\% $\mathrm{BSA}$ ) for $2 \mathrm{~h}$ at room temperature. To label F-actin with a fluorescent probe, the cells were incubated with fluorescein isothiocyanate-phalloidin ( $5 \mu \mathrm{g} / \mathrm{ml}$; cytoskeleton) for $30 \mathrm{~min}$ at room temperature. The fluorescently labeled cells were subsequently observed and imaged by confocal microscopy (Leica TCS SP5 II, Leica Microsystems CMS GmbH). The fluorescence intensity of the labeled vinculin and F-actin in each cell was quantified by analysis of the fluorescent confocal microscopy images, using Image-Pro Plus software (Media Cybernetics, Inc., Rockville, MD, USA).

Correlation analysis and statistics. Pearson's correlation was tested using the Statistical Product and Service Solution v.17 (SPSS, Inc., Chicago, IL, USA). Significant differences were analyzed using one-way analysis of variance followed by Tukey's test for multiple comparisons between groups, using SigmaPlot 12.0 (SysStat Software, San Jose, CA, USA). P<0.05 was considered to indicate a statistically significant difference.

\section{Results}

OVA sensitization induces ADAM33 protein expression. Fig. 1 demonstrates the levels of the protein expression of ADAM33 in ASMCs from rats recieving OVA sensitization for 4, 6 or 12 weeks, and from the controls, as quantified by western blot analysis. Fig. 1A shows the western blot image in which an intense band of $\sim 55 \mathrm{kDa}$, corresponding to the molecular weight of ADAM33 protein was observed, indicating ADAM33 expression. Lanes 1-4 in Fig. 1A represent samples from the controls and the rats sensitized by OVA for 4, 6 and 12 weeks, respectively. Fig. 1B shows the quantitative results of the western blot analysis, as defined by the ratio of the expression of ADAM33 protein to that of $\beta$-tubulin. Compared with the controls, the protein expression of ADAM33 was increased in all ASMCs from the OVA-sensitized rats, and this increase peaked when the rats were sensitized for 4 weeks and then gradually declined as OVA sensitization continued $(\mathrm{P}<0.001)$.

OVA sensitization leads to stiffening, but no change in contractility, of the ASMCs. Fig. 2 demonstrates the cell stiffness and contractility of ASMCs from rats with or without OVA sensitization, as measured by OMTC. Fig. 2A shows the log-log plot of the cell stiffness $\left(G^{\prime}\right)$ versus the frequency of measurement $(0.1-100 \mathrm{~Hz})$. In all cases, the cell stiffness exhibited typical power-law behavior, in which cell stiffness increased with frequency $(f)$ as a power-law response $\left(G^{\prime} \sim f^{\alpha}\right)$, where $\alpha$ is the power-law exponent that is typically between
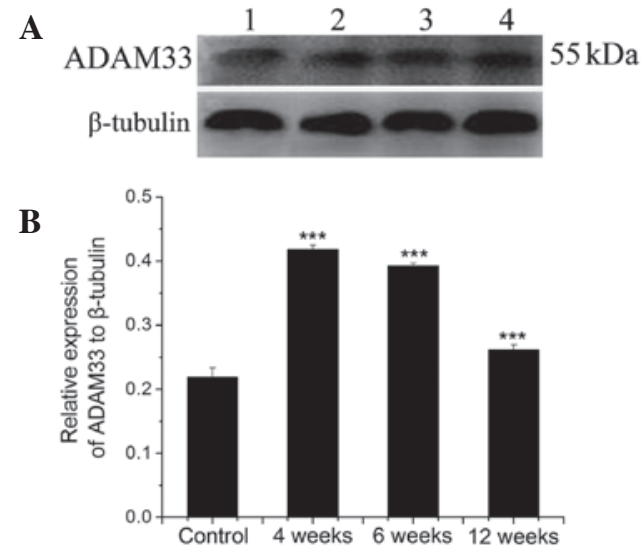

Figure 1. OVA sensitization induced the time-dependent increase in ADAM33 protein expression in ASMCs. (A) The relative expression of the ADAM33 protein to $\beta$-tubulin in the ASMCs from non-sensitized SD rats (controls) or rats receiving OVA sensitization for 4, 6 and 12 weeks, respectively (lanes 1-4). (B) The ratio of the expression of ADAM33 protein to that of $\beta$-tubulin. In all cases, the level of protein expression of ADAM33 in ASMCs from OVA-sensitized rats was significantly increased compared with that of the controls; however, the peak of expression occured when the rats were sensitized for 4 weeks. Data are presented as the mean $\pm \mathrm{SD}$. $\left({ }^{* * *} \mathrm{P}<0.001\right.$, $\mathrm{n}=5$ ). OVA, ovalbumin; ADAM33, a disintergrin and metalloproteinase 33; ASMCs, airway smooth muscle cells.

0.1 and 0.3 for living cells (20). The magnitude of the cell stiffness was observed to be in the ascending order of 12 weeks, control, 6 weeks and 4 weeks, and $\alpha$ also increased with the same trend. This trend was further demonstrated by the cell stiffness measured at a single constant frequency $(0.3 \mathrm{~Hz})$, the $\mathrm{G}_{0}$ of ASMCs from rats with or without OVA sensitization. Compared with the control rats, $\mathrm{G}_{0}$ in the OVA-sensitized rats significantly increased, peaked when the rats were sensitized for 4 weeks $(\mathrm{P}<0.05)$ and gradually declined with continued sensitization (Fig. 2B).

Fig. 2C shows a typical time course plot of cell stiffness in response to $\mathrm{KCl}$ stimulation. Prior to the addition of $\mathrm{KCl}$, the cell stiffness fluctuated with an averaged baseline level $\left(\mathrm{G}_{0}\right)$. Following the addition of $\mathrm{KCl}$ to the ASMCs, the cells responded by contraction that rapidly increased the cell stiffness to a higher level $\left(\mathrm{G}_{\mathrm{KCl}}\right)$ in $\sim 1 \mathrm{~min}$. Thus, the contractility of ASMCs with $\mathrm{KCl}$ stimulation was quantified as the ratio between the cell stiffness following $\mathrm{KCl}$ activation $\left(\mathrm{G}_{\mathrm{KCl}}\right)$ and the baseline cell stiffness $\left(\mathrm{G}_{0}\right)$, i.e. $\mathrm{G}_{\mathrm{KCl}} / \mathrm{G}_{0}$. As demonstrated in Fig. 2D, OVA sensitization did not result in a significant change in the contractility of ASMCs from SD rats.

OVA-sensitization results in an acute increase in the traction force of ASMCs. Fig. 3 demonstrates the effect of OVA sensitization on the traction force generated by ASMCs. Fig. 3A shows the phase-contrast image of a single ASMC cultured on the polyacrylamide substrate, and Fig. 3B demonstrates the field of the traction force generated in this cell. From the traction force field, the net contractile moment of each ASMC was quantified. In Fig. 3C, it was observed that compared with the controls, the traction force generated by ASMCs was significantly greater in the rats that had been sensitized by OVA for 4 weeks $(\mathrm{P}<0.05)$, and marginally (but not significantly) enhanced for the longer duration of OVA sensitization. 

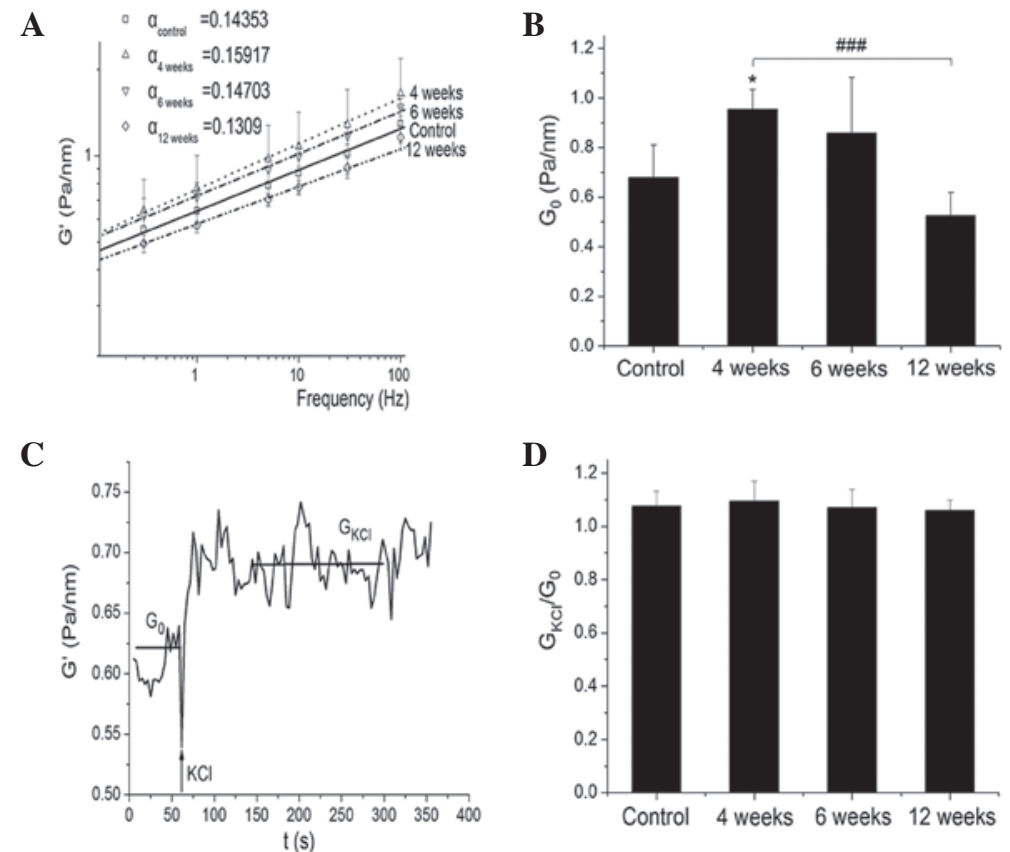

Figure 2. Stiffness and contractility of the ASMCs from either non-sensitized controls or OVA-sensitized rats. (A) The stiffness of the ASMCs measured by OMTC across the spectrum of frequencies $(0.1-100 \mathrm{~Hz}$ ). Data on the ASMCs from rats that were either non-sensitized (controls, $\square)$ or OVA-sensitized for $4(\Delta)$, $6(\nabla)$ or 12 weeks $(\diamond)$. The straight lines represent the linear-fitting for the log-log plots of the cell stiffness $\left(\mathrm{G}^{\prime}\right)$ versus the frequency, displaying power-law relationships with corresponding slopes $(\alpha)$. (B) Baseline stiffness $\left(\mathrm{G}_{0}\right)$ measured at a constant $0.3 \mathrm{~Hz}$ and averaged over $\sim 50$ sec for ASMC from either nonsensitized (controls) or OVA-sensitized rats (from left to right, 4, 6 and 12 weeks, respectively). (C) A typical time course of ASMC measured by OMTC at a constant $0.3 \mathrm{~Hz}$ prior to and following the addition of $\mathrm{KCl}\left(80 \mathrm{mM}\right.$; isotonic). Prior to the addition of $\mathrm{KCl}$, the stiffness fluctuated about the baseline level $\left(\mathrm{G}_{0}\right)$. Upon addition of $\mathrm{KCl}(\sim 6 \mathrm{sec})$, ASMCs contracted and thus the stiffness rapidly increased from the baseline level $\left(\mathrm{G}_{0}\right)$ and stabilized at a higher level $\left(\mathrm{G}_{\mathrm{KCl}}\right)$ in $\sim 1 \mathrm{~min}$. (D) ASMC contractility quantified as the ratio of the stiffness measured following the addition of $\mathrm{KCl}$ to the baseline stiffness measured prior to the addition of $\mathrm{KCl}\left(\mathrm{G}_{\mathrm{KC} /} / \mathrm{G}_{0}\right)$. The data bars from left to right represent the quantified contractility of ASMC from rats that were either non-sensitized (controls), or OVA-sensitized for 4, 6 and 12 weeks, respectively. ("P<0.05 and $\left.{ }^{\# \# \# ~} \mathrm{P}<0.001, \mathrm{n}=6\right)$. OVA, ovalbumin; ASMCs, airway smooth muscle cells; OMTC, optical magnetic twisting cytometry.

A

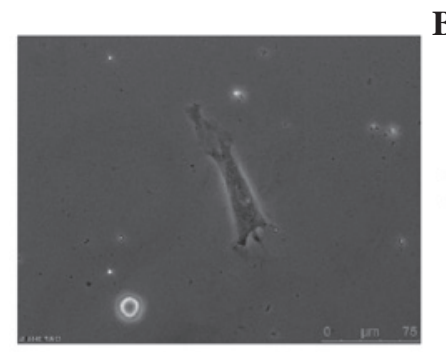

B
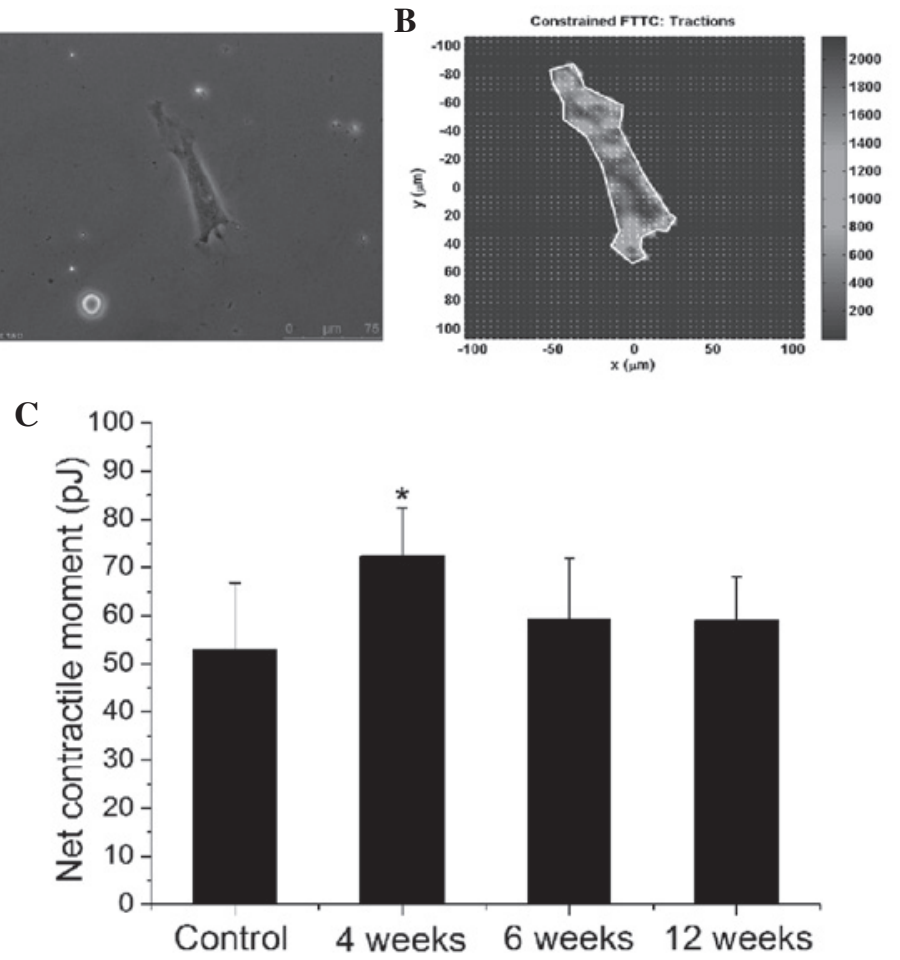

Figure 3. Traction force generated by the ASMCs from control and OVA-sensitized rats. (A) A respresentative phase-contrast microscopic image of a single ASMC cultured on polyacrylamide substrate. (B) A map of traction force generated by the same cell shown in (A), which was measured by Fourier transform traction microscopy. (C) Net contractile moment computed from the traction force map as shown in (B). The data bars from left to right represent the quantified net contractile moments of ASMC from rats that were either non-sensitized (controls), or OVA-sensitized for 4, 6 and 12 weeks, respectively. ("P<0.05, $\mathrm{n}=9$ ). OVA, ovalbumin; ASMCs, airway smooth muscle cells. 
A
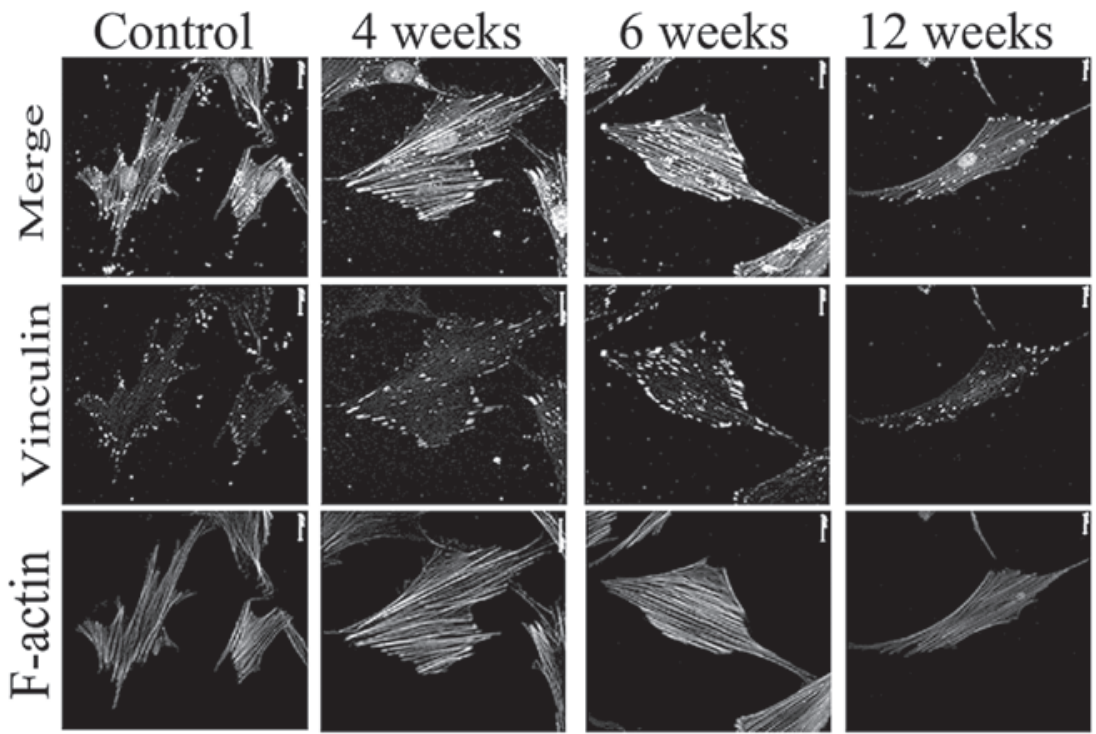

B

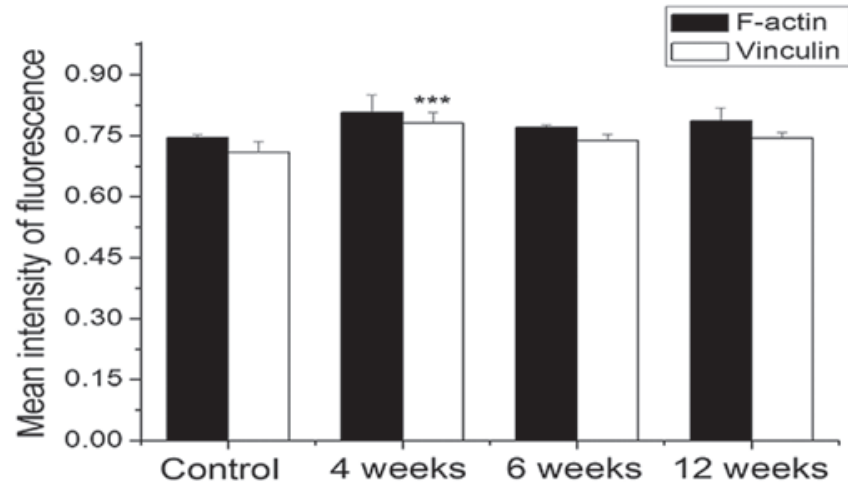

Figure 4. Remodeling of the cytoskeletal structure of ASMCs in response to OVA sensitization. (A) Representative images of the ASMC cytoskeleton characterized by fluorescently labeled vinculin (middle row panel) and F-actin (lower row panel), and visualized by confocal microscopy (magnification, x63; oil). The column panels from left to right represent ASMC from rats that were either non-sensitized (controls) or OVA-sensitized for 4, 6 and 12 weeks, respectively. The upper row panel displays merged corresponding vinculin and F-actin images. (B) Mean expression of vinculin and F-actin in the ASMCs was quantified as the fluorescence intensity of labeled vinculin and F-actin averaged over the area of the ASMC. The data bars from left to right represent the quantified mean expression of F-actin (solid bar) and vinculin (open bar) in ASMCs from rats that were either non-sensitized (controls) or OVA-sensitized for 4, 6 and 12 weeks, respectively $\left({ }^{* * * *} \mathrm{P}<0.001, \mathrm{n}=5\right)$. OVA, ovalbumin; ASMCs, airway smooth muscle cells.

Table I. Cp between ADAM33 protein expression and the G', traction force, vinculin expression and F-actin expression of ASMC.

\begin{tabular}{lcccc}
\hline Cp & G' & $\begin{array}{c}\text { Traction } \\
\text { force }\end{array}$ & Vinculin & F-actin \\
\hline ADAM33 & 0.864 & 0.716 & 0.774 & 0.662 \\
\hline
\end{tabular}

Cp, Pearson's correlation coefficients; ADAM33, a disintegrin and metalloproteinase 33; G', stiffness; ASMC, airway smooth muscle cells.

OVA sensitization enhances vinculin and F-actin expression in ASMCs. Fig. 4 demonstrates the effect of OVA-sensitization on the expression of vinculin and F-actin in the ASMCs of the SD rats. Representative images of vinculin and F-actin, labeled by respective fluorescent probes and visualized by laser scanning confocal microscopy, are shown in the middle and lower panels of Fig. 4A. The merged results of vinculin and F-actin images are shown in the top panel of Fig. 4A. Images from the left to the right of Fig. 4A represent the cytoskeletal structure of the ASMCs in control rats or in rats sensitized by OVA for 4, 6 and 12 weeks, respectively. Through the analysis of the fluorescence intensity in the images, it appears that ASMCs from the OVA-sensitized rats exhibited marginally higher levels of fluorescence intensity for vinculin and F-actin compared with those of the controls. In particular, ASMCs from the rats sensitized by OVA for 4 weeks exhibited the greatest level of vinculin expression $(\mathrm{P}<0.001)$.

Correlation between ADAM33 protein expression and the mechanics of ASMCs. The similarity between the OVA-induced ADAM33 protein expression and the altered mechanical properties of ASMCs in response to OVA sensitization suggested that the protein expression of ADAM33 was correlated with the mechanics of the ASMCs. Further analysis of these results with respect to Pearson's correlation coefficient resulted in largely positive coefficients between the tested results of ADAM33 protein expression and the measured 
mechanical properties, including the cell stiffness, traction force, and levels of vinculin and F-actin (Table I).

\section{Discussion}

The present study demonstrated that OVA sensitization of SD rats resulted in increased protein expression levels of ADAM33 in the ASMCs, in a time-dependent manner. The protein expression levels of ADAM33 in the ASMCs reached a maximum at $\sim 4$ weeks of OVA sensitization, and then gradually subsided as OVA sensitization continued. Notably, the mechanical properties of the ASMCs, including cell stiffness and traction force, were altered during the course of OVA sensitization in the same manner as that of the ADAM33 protein. The OVA-induced alteration of the ASMC mechanics and ADAM33 protein expression were positively correlated. These results implied that ADAM33 protein expression was correlated with the mechanics of the ASMCs during the pathogenesis and progression of asthma.

The protein expression of ADAM33 was increased in the ASMCs of the OVA-sensitized rats, which was consistent with a previous study that demonstrated that the expression of ADAM33 was increased in asthmatic patients (1). However, in this study, ASMCs were studied in vitro, and the protein expression of ADAM33 was likely to be variable as the primary cells were passaged over time (21). To control for this passagedependent variability, only the cells between passages 2 to 5 were used in the present study, and the relative expression of the ADAM33 protein was obtained from cells of the same passage.

ASMC mechanics are generally recognized as the final pathway to AHR that characterizes asthma $(11,13)$. In the present study, it was demonstrated that in the OVA-sensitized SD rat models of asthma, the magnitude of ASMC stiffness increased during OVA sensitization. Notably, the time dependence of the cell stiffness of the ASMCs appeared to be similar to that of the protein expression of ADAM33. Statistical analysis verified that the stiffness was positively correlated with the ADAM33 protein expression in the ASMCs of the OVA-sensitized rats (Pearson's correlation coefficient, 0.864). ASMC stiffness also exhibited characteristic power-law behavior when measured over a range of frequencies, which was consistent with previous studies $(15,22)$. However, it was noted that the contractility of the ASMCs as determined by the stiffness increase in response to $\mathrm{KCl}$ stimulation was not affected by OVA sensitization. The absence of an effect of OVA-sensitization on the KCl-induced contractility in the ASMCs was somewhat but not completely surprising, due to the nature of $\mathrm{KCl}$ stimulation. As a non-specific contractile agonist, it is possible that the OVA sensitization was only effective on certain structure(s) of the contractile machinery of the ASMCs, which $\mathrm{KCl}$ was unable to target or activate. Specific agonists, such as histamine, that target the G-protein coupled receptors may be used to further investigate this.

Fourier transform traction microscopy was utilized to assess the ASMCs for the ability to generate traction force at the single-cell level. The results demonstrated that compared with the non-sensitized rats, ASMCs from the OVA-sensitized rats generated greater traction force, and the extent of traction force enhancement was also positively correlated with the increase in ADAM33 protein expression during OVA sensitization (Pearson's correlation coefficient, 0.716). The enhancement of traction force generation by the ASMCs may be attributed to various factors, including a strengthened cytoskeletal structure, the interaction of actin and myosin filaments (19) and enhanced focal adhesions due to an increased quantity of structural and signaling proteins, such as vinculin (23). These factors, separately or combined, may enhance the physical links between the cytoskeleton and the extracellular matrix, exerting a greater traction force to the extracellular matrix (24). In the present study, the results of the confocal microscopy of the ASMCs that were fluorescently labeled for vinculin and F-actin demonstrated that the ASMCs from the OVA-sensitized rats exhibited a greater level of fluorescence intensity of vinculin and F-actin compared with those from the non-sensitized rats. The increases in vinculin and F-actin were also positively correlated with the increased protein expression of ADAM33 during OVA-sensitization (Pearson's correlation coefficients, 0.774 and 0.662 for vinculin and F-actin, respectively). This suggests a possible signaling pathway of ASMCs in which ADAM33 protein modulates cell mechanics via the regulation of cytoskeletal proteins, such as vinculin and F-actin.

Furthermore, the results demonstrated that the effects of OVA sensitization on the ASMCs were greatest when the rats were sensitized for 4 weeks, and then they gradually decreased regardless of the continuation of OVA sensitization. This time dependence of the efficacy of OVA sensitization may be largely attributed to the phenomenon of desensitization, which has been observed in repeatedly challenged rats and guinea pigs $(25,26)$. The rate of desensitization was faster for ASMC mechanics compared with that of the expression of the ADAM33 protein. This difference implied that the ASMC mechanics that characterize airway function may be more sensitive and adaptable to the presence of an allergen compared with biological targets, such as ADAM33 protein (27); however, further studies are required.

To the best of our knowledge, this study demonstrated for the first time in vivo that OVA sensitization of SD rats led to the increased expression of ADAM33 protein in ASMCs. OVA sensitization also resulted in increased stiffness, traction force and expression of vinculin and F-actin in ASMCs. The extent of these changes were time-dependent during OVA sensitization. Notably, the change in the expression of ADAM33 protein in the ASMCs was correlated with the changes in ASMC mechanics, including cell stiffness, traction force and the expression of vinculin and F-actin. This suggested that the ADAM33 protein and the ASMC mechanics may be concordantly involved in the pathogenesis and progression of asthma. As ADAM33 is an asthma susceptibility gene and ASMC mechanics are the common pathway to AHR that is the hallmark of asthma, these results may have identified a potential mechanism through which ADAM33 contributes to the pathogenesis of asthma, and thus may aid in the development of novel ADAM33-based therapeutics to treat asthma.

\section{Acknowledgements}

This study was supported by the National Natural Science Foundation of China (grant no. 11172340); the Training Program for Hundreds of Distinguished Leading Scientists 
of Chongqing, Chongqing Natural Science Foundation (project nos. CSTC2010BA5001 and CSTC2012jjA0588); the Fundamental Research Funds for the Central Universities (grant no. CQDXWL-2012-123); the Specialized Research Fund for the Doctoral Program of Higher Education of China (grant no. 20120191120032); and the Fundamental Research Funds for the Central Universities (project nos. CDJXS11230038 and CDJXS11230027).

\section{References}

1. Lee JY, Park SW, Chang HK, et al: A disintegrin and metalloproteinase 33 protein in patients with asthma: Relevance to airflow limitation. Am J Respir Crit Care Med 173: 729-735, 2006.

2. Rogers DF: Airway mucus hypersecretion in asthma: an undervalued pathology? Curr Opin Pharmacol 4: 241-250, 2004.

3. Zhang Y, Moffatt MF and Cookson WO: Genetic and genomic approaches to asthma: new insights for the origins. Curr Opin Pulm Med 18: 6-13, 2012.

4. Lee JH, Park HS, Park SW, et al: ADAM33 polymorphism: association with bronchial hyper-responsiveness in Korean asthmatics. Clin Exp Allergy 34: 860-865, 2004.

5. Tripathi P, Awasthi S, Prasad R, et al: Association of ADAM33 gene polymorphisms with adult-onset asthma and its severity in an Indian adult population. J Genet 90: 265-273, 2011.

6. Van Eerdewegh P, Little RD, Dupuis J, et al: Association of the ADAM33 gene with asthma and bronchial hyperresponsiveness. Nature 418: 426-430, 2002.

7. Holgate ST, Davies DE, Powell RM and Holloway JW: ADAM33: a newly identified protease involved in airway remodelling. Pulm Pharmacol Ther 19: 3-11, 2006.

8. Werner M, Herbon N, Gohlke H, et al: Asthma is associated with single-nucleotide polymorphisms in ADAM33. Clin Exp Allergy 34: 26-31, 2004.

9. Powell RM, Wicks J, Holloway JW, et al: The splicing and fate of ADAM33 transcripts in primary human airways fibroblasts. Am J Respir Cell Mol Biol 31: 13-21, 2004.

10. Umland SP, Garlisi CG, Shah H, et al: Human ADAM33 messenger RNA expression profile and post-transcriptional regulation. Am J Respir Cell Mol Biol 29: 571-582, 2003.

11. An SS, Bai TR, Bates JH, et al: Airway smooth muscle dynamics: a common pathway of airway obstruction in asthma. Eur Respir J 29: 834-860, 2007.
12. Jie Z, Jin M, Cai Y, et al: The effects of Th2 cytokines on the expression of ADAM33 in allergen-induced chronic airway inflammation. Respir Physiol Neurobiol 168: 289-294, 2009.

13. Song A, Liao Q, Li J, et al: Chronic exposure to sulfur dioxide enhances airway hyperresponsiveness only in ovalbumin-sensitized rats. Toxicol Lett 214: 320-327, 2012.

14. Hirst SJ: Airway smooth muscle cell culture: application to studies of airway wall remodelling and phenotype plasticity in asthma. Eur Respir J 9: 808-820, 1996.

15. Fabry B, Maksym GN, Shore SA, et al: Selected contribution: time course and heterogeneity of contractile responses in cultured human airway smooth muscle cells. J Appl Physiol 91: 986-994, 2001.

16. Fairbank NJ, Connolly SC, Mackinnon JD, et al: Airway smooth muscle cell tone amplifies contractile function in the presence of chronic cyclic strain. Am J Physiol Lung Cell Mol Physiol 295: L479-L488, 2008.

17. Chen C, Krishnan R, Zhou E, et al: Fluidization and resolidification of the human bladder smooth muscle cell in response to transient stretch. PLoS One 5: e12035, 2010.

18. Butler JP, Tolić-Nørrelykke IM, Fabry B and Fredberg JJ: Traction fields, moments, and strain energy that cells exert on their surroundings. Am J Physiol Cell Physiol 282: C595-C605, 2002.

19. Wang JH and Lin JS: Cell traction force and measurement methods. Biomech Model Mechanobiol 6: 361-371, 2007.

20. Deng L, Trepat X, Butler JP, et al: Fast and slow dynamics of the cytoskeleton. Nat Mater 5: 636-640, 2006.

21. Tribius S, Pidel A and Casper D: ATM protein expression correlates with radioresistance in primary glioblastoma cells in culture. Int J Radiat Oncol Biol Phys 50: 511-523, 2001.

22. Fabry B, Maksym GN, Butler JP, et al: Scaling the microrheology of living cells. Phys Rev Lett 87: 148102, 2001.

23. Burton K, Park JH and Taylor DL: Keratocytes generate traction forces in two phases. Mol Biol Cell 10: 3745-3769, 1999.

24. Wang N, Tolić-Nørrelykke IM, Chen J, et al: Cell prestress. I. Stiffness and prestress are closely associated in adherent contractile cells. Am J Physiol Cell Physiol 282: C606-C616, 2002.

25. Andrew DK, Schellenberg RR, Hogg JC, et al: Physiological and immunological effects of chronic antigen exposure in immunized guinea pigs. Int Arch Allergy Appl Immunol 75: 208-213, 1984.

26. Szelenyi I: Animal models of bronchial asthma. Inflamm Res 49: 639-654, 2000

27. Trepat X, Deng L, An SS, et al: Universal physical responses to stretch in the living cell. Nature 447: 592-595, 2007. 